\title{
ZeBox: A novel non-intrusive continuous-use technology to trap and kill airborne microbes
}

\author{
Kruttika S. Phadke ${ }^{1,2}$, Deepak G. Madival ${ }^{1,3}$, Janani \\ Venkataraman $^{1}$, Debosmita Kundu ${ }^{1}$, K. S. Ramanujan ${ }^{1}$, Nisha \\ Holla $^{1}$, Jaywant Arakeri ${ }^{3}$, Gaurav Tomar ${ }^{3}$, Santanu Datta ${ }^{1,4}$, and \\ Arindam Ghatak *1 \\ ${ }^{1}$ Biomoneta Research Private Limited, Bangalore, India 560065 \\ ${ }^{2}$ Department of Veterinary Microbiology and Preventive Medicine, Iowa State \\ University, Ames, IA, 50011, USA \\ ${ }^{3}$ Mechanical engineering department, Indian Institute of Science, Bangalore, India \\ 560012 \\ ${ }^{4}$ Bugworks Research, Bangalore, India 560066
}

April 16, 2021

Abstract $=150$ words $($ limit $=150)$

Introduction (517) + Results $(2141)+$ Discussion $(394)=3052$ words $($ limit $=3000)$

Figures $(6)+\operatorname{Tables}(2)=8($ limit $=7)$

References $=53($ limit $=50)$

\begin{abstract}
1 Abstract
2 Preventing nosocomial infection is a major unmet need of our times. Existing

3 air decontamination technologies suffer from demerits such as toxicity of

4 exposure, species specificity, noxious gas emission, environment-dependent

5 performance and high power consumption. Here, we present a novel

6 technology called "ZeBox" that transcends the conventional limitations and

7 achieves high microbicidal efficiency. In ZeBox, a non-ionizing electric field

8 extracts naturally charged microbes from flowing air and deposits them on

- engineered microbicidal surfaces. The surface's three dimensional topography

10 traps the microbes long enough for them to be inactivated. The electric field

11 and chemical surfaces synergistically achieve rapid inactivation of a broad

12 spectrum of microbes. ZeBox achieved near complete kill of airborne microbes

13 in challenge tests (5-9 log reduction) and $>90 \%$ efficiency in a fully functional

14 stem cell research facility in the presence of humans. Thus, ZeBox fulfills the

15 dire need for a real-time, continuous, safe, trap-and-kill air decontamination

16 technology.
\end{abstract}

*Corresponding author: arindam@biomoneta.com 


\section{${ }_{17} 1$ Introduction}

Microbial load (bacteria, viruses, spores and fungi) in our living, working and hospital space must be reduced to mitigate the transmission of airborne infections. As per CDC (Center for Disease Control, USA)'s recommendation (https://www.cdc.gov/niosh/topics/hierarchy/default.html), eliminating microbes at the source as and when produced is the first line of defense against spread of infections. Filtration, electrostatic precipitation, bactericidal gas spraying, ultra-violet germicidal irradiation (UVGI, employing $\sim 254 \mathrm{~nm}$ radiation), plasma discharge and photo-catalytic oxidation (PCO) are the currently available air decontamination technologies [1]. While some are microbicidal, others only trap microbes. Filtration 2 and electrostatic precipitation [3] belong to the latter category. Microbes trapped inside filters can multiply in situ 4, 5, 6, 7, 8, such filters are detrimental to indoor air quality and hazardous during their disposal. They also offer high flow resistance which translates to high operating power consumption [9, 10]. Electrostatic precipitation uses electric field to attract and trap aerosols pre-charged by corona discharge, but which produces noxious gases like ozone [3, 11. Its microbicidal action is dubious; in fact electrostatic bioaerosol samplers capture microbes that remain viable [12, 13, 14]. However, because of its low flow resistance, it consumes less power per unit of clean air delivered compared to filtration [3. Filters made of anti-bacterial fibers have also been developed [15, 16, 17, 18, 19, 20] but their performance remains be proven under realistic indoor conditions.

Bactericidal gas spraying, UVGI, plasma discharge and PCO are microbicidal technologies. Although bactericidal gases and UVGI can sterilize an entire room, they cannot be deployed in human presence. UVGI is used to sterilize upper room air and air circulating through ventilation ducts.

However, microbicidal action of UVGI depends on environmental parameters such as humidity [21, 22, 23], is species-specific [24] and requires a minimum duration of exposure to microbes 25. Exposure of humans to UVGI (due to faulty design, deployment or use of UVGI devices) can damage their eyes and skin [26, 27, 28, 29]. UVGI is used to kill microbes trapped on a filter's surface [30, 31] but then it cannot reach microbes residing beneath the surface.

Plasma discharge [32] and PCO [34, 35] both generate ions and/or reactive species, respectively using gas discharge and reaction with an irradiated catalyst. However, they also generate $\mathrm{NO}_{X}$ and ozone [1] and additional methods are necessary to mitigate them 33. In PCO, convection of gas to the catalyst and the subsequent adsorption, reaction and release of reactive species into the bulk flow is the bottleneck process [36, which results in low clean air delivery rates [1].

Given the importance of eliminating airborne infection, a technology that is safe, suitable for continuous use and efficient against a wide variety of airborne microbes is desirable. Here, we describe such a novel technology called "ZeBox"; the name derives from the Zeta-potential possessed by microbes, which property is pivotal in trapping them inside the Box-shaped device. In the following, we discuss the working mechanism of ZeBox and demonstrate its efficacy in chamber tests and field studies against a variety of microbes. 


\section{Results}

\section{Electrode plates with engineered chemical surfaces form the kill}

cassette. A row of flat plate electrodes $(10.9 \mathrm{~cm} \times 30 \mathrm{~cm})$ with alternating polarity are assembled inside a cuboid shaped box with open ends for transmitting flow. A three dimensional hydrocellular microbicidal composite material (US patent no. US $9566363 \mathrm{~B} 2$, licensed) is layered on to the electrodes. A non-ionizing $3 \mathrm{kV} / \mathrm{cm}$ electric field is set up between electrodes by applying direct-current voltage between them. Microbes are trapped and killed inside this "kill cassette". Axial fans pull microbe laden ambient air through the kill cassette and between the electrode-plates, as shown schematically in figure 1 .

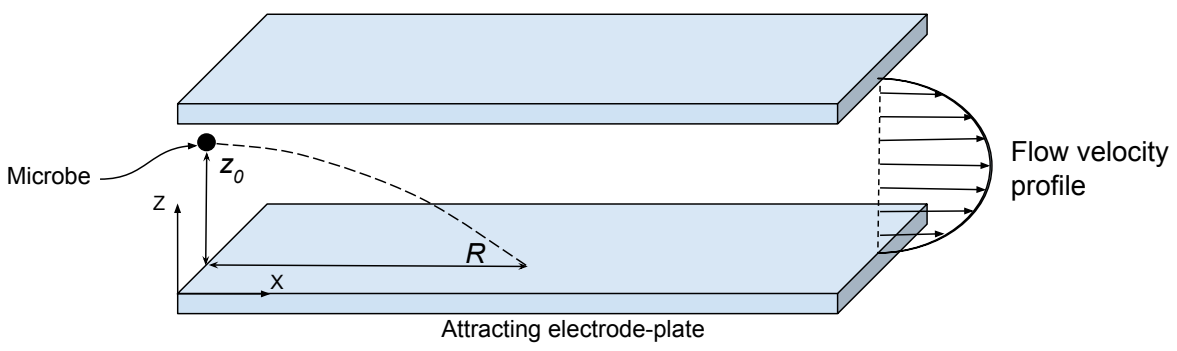

Figure 1: Microbe in a flow subject to transverse electric field. A charged microbe deviates from the flow direction due to the electric field between electrode-plates.

Electric field extracts charged microbes from the flow. Microbes are naturally charged [38, 39]; therefore, in an electric field, they are impelled towards the electrode of opposite polarity. Figure 1 depicts this process schematically. Here, $X$-axis points along the flow and $Z$-axis points away from the attracting electrode. A microbe initially at distance $z_{0}$ from the attracting electrode travels a distance $R$ in the streamwise direction, called its "range", as it descends to $z=0$. Whether or not the microbe hits the electrode depends on its length, the microbe's initial distance $z_{0}$, strength of the electric field, charge on the microbe and the type of flow (laminar or turbulent). The Reynolds number for the flow between electrodes in ZeBox is $\sim 10^{3}$ and a rectangular duct flow (or even plane Poiseuille flow) undergoes transition at this Reynolds number and could be turbulent [40, 41]. Analyzing microbe's motion in a turbulent flow is difficult because of its complicated, stochastic nature; supplementary information S1 analyzes microbe's motion and its maximum range in a laminar flow instead.

Earlier studies on resuspension of dust from flat surfaces due to a flow show that, whenever the hydrodynamic force and torque exerted by the flow exceed those that keep the particles attached to the surface (for example, Van der Waals force), the particles can either detach by lifting off or slide and roll on the surface [42, 43]. In our case, lifting off of microbes from the electrode is unlikely due to the strong electric field, but they can nevertheless slide and roll and thus escape away due to the electrode's finite length (refer figure 2). Since the microbicidal surface requires a minimum duration of contact to inactivate 


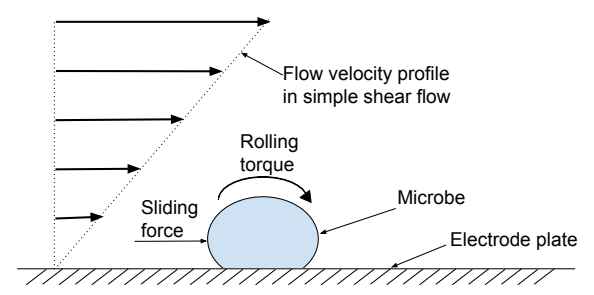

Figure 2: Microbe sliding and rolling on a flat surface. Microbes can slide and roll over a flat surface due to hydrodynamic force and torque exerted by the flow.

microbes depending on how sensitive or hardy it is, a fraction of the deposited microbes could escape while still viable. Therefore, the ability of the microbicidal surface to trap and hold microbes until they are inactivated becomes important.

\section{Three dimensional topography of the microbicidal surface traps the} microbe. The microbicidal surface employed in ZeBox has a highly uneven topography at the microbial scale, populated with well-like depressions to trap and hold microbes. Figures $3 \mathrm{a}$ and $3 \mathrm{~b}$ show the scanning electron microscope (SEM) images of the surface at different magnifications appropriate to the microbial scale. Figure 3c shows streamlines in a numerically simulated two dimensional flow (using OpenFOAM-7) over a surface with square shaped wells, to qualitatively illustrate the kind of flow obtained over an uneven topography. A simple shear flow was imposed on the flow domain (refer figure 3c) by moving its uppermost boundary horizontally at constant speed. The flow Reynolds number based on the imposed shear rate and the dimension of the square-shaped well is $\sim 10^{-5}$, which is appropriate to the flow in the neighborhood of the microbicidal surface in ZeBox. The important feature of the flow for our purpose is the recirculating region set up within the wells, in which the streamlines of the flow form closed loops. This feature is quite general for a flow over an uneven topography and which presumably enhances the efficacy of the microbicidal surface further in regard to trapping microbes. Once the microbe falls into one of the wells, brought there either in the course of its rolling over the surface or directly by the electric field, the recirculating flow can confine it to the well for a sufficiently long duration.

Table 1 shows the efficacy of microbicidal surfaces (in terms of $\log _{10}$ reduction, where $n$ - $\log _{10}$ reduction implies reduction in the initial microbial load by a factor of $10^{n}$ ) with different topographies, which we call 2-D and 3-D surfaces, in flow experiments. A 2-D surface is a single layer of cotton fabric while a 3-D surface is a multilayered 90:10 polyethylene : cotton fabric. In the presence of electric field, 3-D microbicidal surface performs better than the 2-D surface. When the electric field is absent, the microbes are not extracted from the flow and hence both surfaces perform similarly.

\section{Electric field and chemical microbicidal-surfaces synergistically} achieve rapid inactivation of microbes. In contrast to electrostatic precipitators, the applied electric field in ZeBox plays two roles: it pulls 

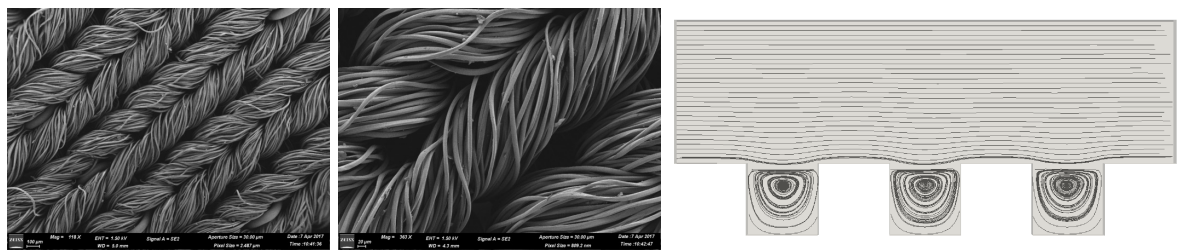

(a) $118 \times$ magnifica- (b) $363 \times$ magnifica- (c) Recirculating flow due to uneven tion. tion. topography.

Figure 3: Topography of the microbicidal surface at microbial scale. SEM photographs revealing the highly uneven topography of microbicidal surface and the expected flow patterns over it.

\begin{tabular}{|r|c|c|}
\hline & \multicolumn{2}{|c|}{ Microbial load reduction $\left(\log _{10}\right.$ scale) } \\
\cline { 2 - 3 } & 3 -D surface & 2 -D surface \\
\hline Without electric field & $2.82^{ \pm 0.74}$ & $2.13^{ \pm 0.2}$ \\
\hline With electric field & $9.42^{ \pm 1.02}$ & $4.68^{ \pm 0.88}$ \\
\hline
\end{tabular}

Table 1: Effect of surface topography on microbicidal action. $\log _{10^{-}}$ reduction in viable microbial load (E. coli) achieved by ZeBox with 3-D and 2-D microbicidal surfaces in 10 minutes. Applied electric field $=3 \mathrm{kV} / \mathrm{cm}$. Superscripts show standard deviation.

\begin{tabular}{|r|l|l|}
\hline \multirow{2}{*}{ Time (mins) } & \multicolumn{2}{|c|}{ Microbial load reduction $\left(\log _{10}\right.$ scale) } \\
\cline { 2 - 3 } & With electric field & Without electric field \\
\hline 2 & $3.00^{ \pm 0.39}$ & $0.87^{ \pm 0.44}$ \\
\hline 5 & $5.71^{ \pm 0.19}$ & $1.86^{ \pm 0.78}$ \\
\hline 10 & $8.83^{ \pm 0.69}$ & $2.56^{ \pm 1.17}$ \\
\hline
\end{tabular}

Table 2: Effect of applied electric field on the efficacy of microbicidal surface. Effect of $3 \mathrm{kV} / \mathrm{cm}$ electric field on the $\log _{10}$-reduction in viable microbial load (E. coli) over the microbicidal surface in spot experiments. Superscripts show standard deviation.

microbes from the flow on to the microbicidal surface and then accelerates their subsequent inactivation. Table 2 shows $\log _{10}$-reduction in the microbial load in spot experiments, with $3 \mathrm{kV} / \mathrm{cm}$ electric field applied between electrodes. The microbicidal surface achieves the highest reduction in microbial load in the presence of the electric field. Quaternary ammonium compounds (QAC) are membrane-active agents which inactivate microbes by targeting their cytoplasmic membrane [46, 47, 48, 49], but first, they must breach the outer cell wall. In the present design, QAC is tethered to the 3-D surface by long flexible chains, which presumably helps the QAC to orient itself to puncture holes in the microbe. The external electric field increases the trans-membrane voltage of the cell above its resting value, leading to an electric current that presumably flows through these pores as they form the path of least resistance. This current flow may be analogous to the electroporation of bacteria in which the pores formed in the cell wall are stabilized [50. The intracellular components then leak from the pores, as is 


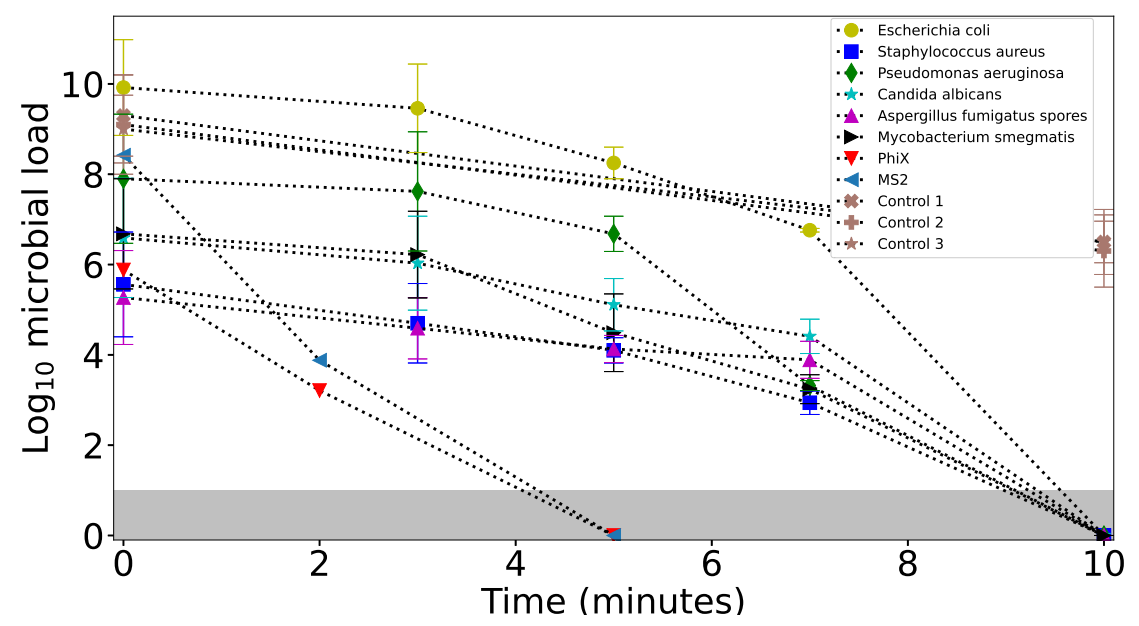

(a) Reduction in microbial load.

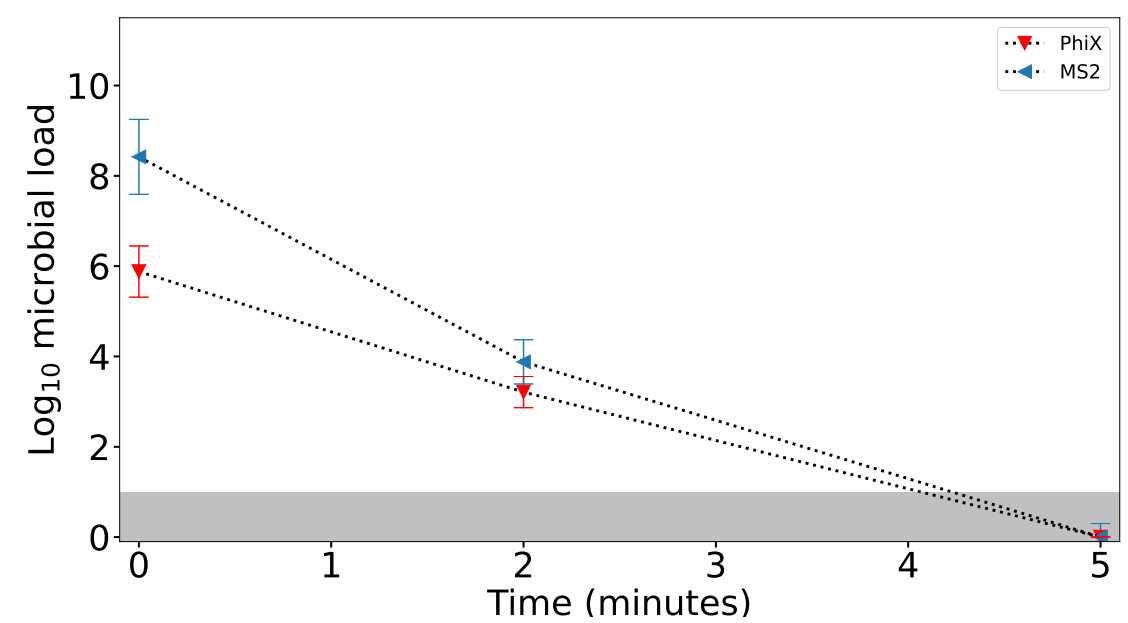

(b) Reduction in viral load.

Figure 4: Reduction of microbial load in test chamber. Variation of the $\log _{10}$ microbial load over time after ZeBox is turned on in the test chamber. The shaded region indicates limit of detection (LoD). Control 1, 2, 3 refer to control experiments employing respectively microbicidal surface without electric field, control surface with electric field and control surface without electric field. 


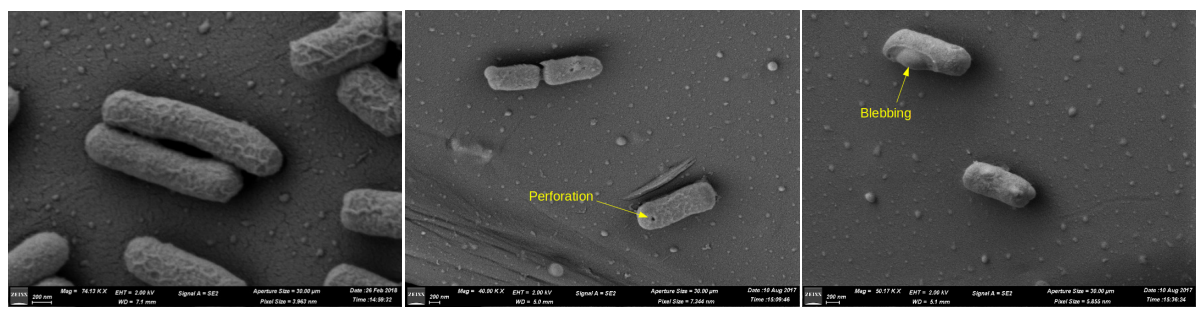

(a) E. coli: control

(b) E. coli: 5 minutes

(c) E. coli: 5 minutes

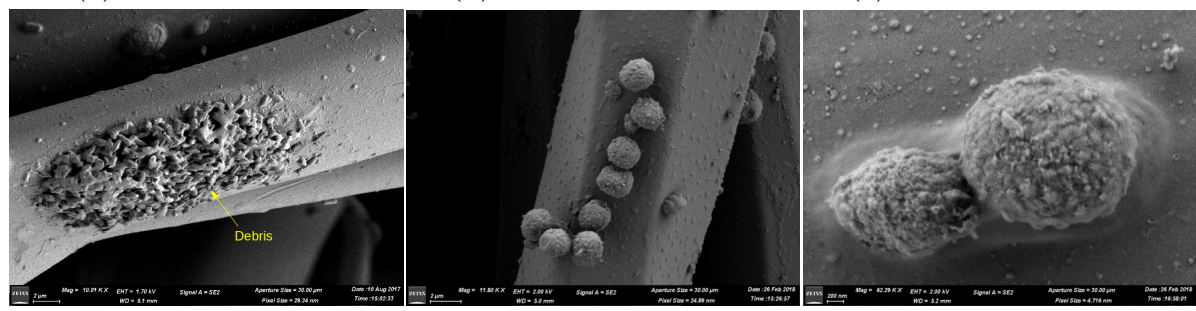

(d) E. coli: 10 minutes(e) A. fumigatus spores:(f) A. fumigatus spores: 15 control minutes

Figure 5: Microbicidal action of the chemical surface. SEM images showing microbes on the microbicidal surface being killed by electroporation.

seen in the SEM pictures. This process leads to the irreversible killing of the cells. Therefore, the chemical surface in tandem with the electric field displays an enhanced electro-chemical microbicidal action compared to what they would have achieved separately.

ZeBox rapidly reduces microbial load in chamber tests. The capability of ZeBox to decontaminate a closed space containing airborne microbes was determined by challenge tests [51]. A broad spectrum of microorganisms was employed in the test - standard gram-positive and gram-negative bacteria of ESKAPE group (Escherichia coli, Staphylococcus aureus, Pseudomonas aeruginosa), mycobacterium species (Mycobacterium smegmatis), fungal species (Aspergillus fumigatus spores and Candida albicans) and virus (PhiX 174 coliphage and MS2 coliphage). Among these, MS2 virus is an accepted surrogate for the SARS-CoV2 virus [52, 53]. Figure 4 shows the collated data on the variation in $\log _{10}$ microbial $\operatorname{load}\left(n-\log _{10}\right.$ microbial load equals $10^{n}$ microbes) over time after ZeBox was turned on. ZeBox proves to be extremely effective in rapidly decreasing the viable microbial load in a closed space. It achieved $9.9 \log _{10}$-reduction (i.e. 99.999999999\% reduction) of $E$. coli in 10 minutes ( $n \log _{10}$-reduction equals reduction by a factor of $10^{n}$ ). For other microbes ZeBox brought about 5 to 9 $\log _{10}$-reduction (i.e. 99.999-99.9999999\% reduction) of the viable microbial load.

SEM images of microbicidal action. Scanning electron microscopy (SEM) studies were done to see how microbes trapped on the microbicidal surface are killed. E. coli and A. fumigatus spores were chosen because they form two extremes on the scale of sensitivity, with spores being hardy. Figure 


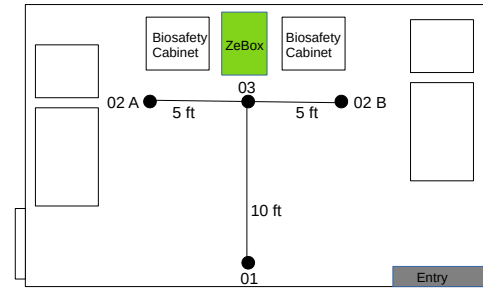

(a) Setup of the field experiment.

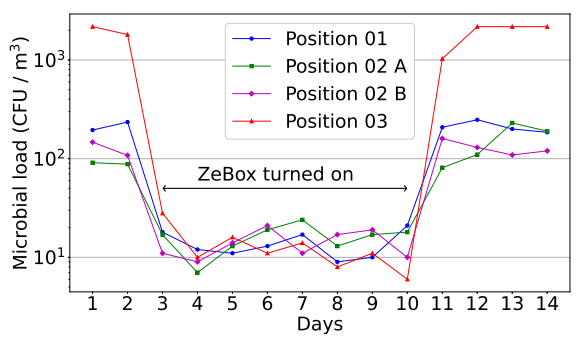

(b) Reduction in microbial load

Figure 6: Field performance of ZeBox. ZeBox reduces the microbial load in an open room. Measurement locations are indicated by filled circles in the schematic.

\begin{abstract}
$5 \mathrm{a}$ and $5 \mathrm{e}$ show the microbes in control conditions. Due to electro-chemical action at the three dimensional microbicidal surface, their cell membrane undergoes morphological changes followed by complete degradation. Figure $5 \mathrm{~b}$ and $5 \mathrm{c}$, obtained after 5 minutes of contact, reveals puncturing and blebbing of the $E$. coli cell membrane. Ultimately, the cells burst and their intracellular contents spill out (figure $5 \mathrm{~d}$ and $5 \mathrm{f}$ ) signaling a complete degradation of the microbes.
\end{abstract}

ZeBox reduces microbial load in open room. ZeBox's performance was also tested in a real life setting, i.e. in a room with constant influx of microbes from outside or due to internal sources. A working tissue culture laboratory in a building with central air-conditioning, but without High Efficiency Particulate Air (HEPA) filters, was chosen for the purpose. Figure 6a shows the schematic plan-view of the lab and the measurement locations. The working people in the lab were the primary source of microbial contamination. Figure $6 \mathrm{~b}$ shows that the microbial load at location-03 where tissue culture work was carried out was $>3000 \mathrm{CFU} / \mathrm{m}^{3}$ initially. ZeBox reduced the microbial load in the lab to $\sim 10 \mathrm{CFU} / \mathrm{m}^{3}$ within about 3 hours after it was turned on. This low level was consistently maintained so long as ZeBox was operational. When it was turned off at day 10, the microbial load rebounded to its original level. During its operation, ZeBox effectively decontaminated a zone of dimensions $\sim 10$ feet $\times 10$ feet (refer figure 6a), which demonstrates its potential to decontaminate a smaller region of interest in a relatively large open room, with continuous movement of personnel and without needing physical partitions.

ZeBox does not produce ozone. Since ZeBox employs non-ionizing electric field, it does not produce ozone (verified in standardized laboratory tests, data not shown here). This is an immense advantage over conventional microbicidal technologies such as plasma and PCO. Also, it consumes $<20$ Watt-hour of electric energy during its operation. 


\section{Discussion and Conclusions}

ZeBox technology exploits the fact that microbes (bacteria, viruses, spores and fungi) are naturally charged and therefore can be readily manipulated by an electric field. Using a non-ionizing electric field, microbicidal surfaces with three dimensional topography and electro-chemical kill mechanism, ZeBox achieves significantly higher microbicidal rate compared to other technologies. Knowing the total reduction in microbial load, as shown in figure 4 , is inadequate to gauge ZeBox's efficacy because any level of decontamination may be achieved given sufficient time. Therefore, an overall microbicidal efficiency must be determined while factoring in the time of operation as well as the volume of the room being decontaminated. Towards this end, we may think in terms of the number of nominal air changes in a room achieved in a given duration and the consequent reduction in microbial load for each air change. In time $t, Q t / V$ number of nominal air changes is achieved, where $Q$ is the air flow rate through ZeBox and $V$ is the volume of the room. If $\eta$ is the corresponding microbicidal efficiency, then $N_{0}$ initial number of viable microbes in the room decreases to $N=N_{0}(1-\eta)^{Q t / V}$ after time $t$. Using this formula and the latest-time data from figure 4 whose ordinate is $\log _{10} N$, we may back-calculate $\eta$ for a specified time duration. The microbicidal efficiency of ZeBox lies in the range of 83-99 \% for all the tests. Considering the variety of sensitive and hardy microbes employed, ZeBox is about equally effective against all of them. Supplementary information S2 provides a theoretical estimation of the microbicidal efficiency of ZeBox and shows that the efficiency deduced from experimental data is aligned with it.

Airborne microbes of size $<2 \mu \mathrm{m}$ can remain suspended in air for several hours before settling down and therefore must be inactivated to reduce the transmission of infections. ZeBox technology presents a universal solution because:

- Freely floating microbes are trapped and killed with high efficiency, eliminating the possibility of future growth.

- The airflow is parallel to antimicrobial surfaces with almost no resistance; therefore, unlike HEPA filters, it has low energy utilization.

- There are no chemical emissions or production of free radicals or ozone; the technology is safe for continuous use in the presence of humans and animals.

- It is equally effective for different varieties of sensitive and hardy microbes.

\section{Materials and methods}

\section{Challenge tests}

A. Test setup An air-sealed test chamber of dimensions $3 \mathrm{ft} \times 4 \mathrm{ft} \times 3 \mathrm{ft}$ (approximately 1000 liters in volume) was built with multiple sampling and nebulization ports. The environmental parameters such as relative humidity and temperature could be monitored using a probe located inside the 
chamber. During experiments, various microorganisms were aerosolized using a 6-jet collision nebulizer (MESA LABS, BGI) into the chamber, and the device efficiency was monitored by collecting and measuring microbial concentration at different time intervals. A second test chamber of dimensions $3 \mathrm{ft}$ x $2.5 \mathrm{ft}$ x $1 \mathrm{ft}$ (approximately 220 liters in volume) placed inside a biosafety cabinet, with similar aerosolization and sampling port configuration, was used for tests with viruses.

B. Cultivation of test microorganisms To validate the efficiency of the decontamination device, Escherichia coli (MTCC 40), Pseudomonas aeruginosa (MTCC 424), Staphylococcus aureus (MTCC 96), Candida albicans (MTCC 584), Aspergillus fumigatus (MTCC 2544), Mycobacterium smegmatis (MTCC 6), MS2 coliphage (ATCC 15597-B1) and PhiX 174 coliphage (ATCC 13706-B1) were used. For growing Escherichia coli, Pseudomonas aeruginosa and Staphylococcus aureus, Luria broth was used. For growing Candida albicans, Potato dextrose broth was used, while for M. smegmatis, Middlebrook 7H9 broth was used. For enumeration of E.coli, samples were plated on Luria Bertani agar; Cetrimide agar was used as a selective for the growth and isolation of Pseudomonas aeruginosa. Cetrimide inhibits the growth of many microorganisms while allowing Pseudomonas aeruginosa to develop typical colonies. For quantification of Staphylococcus, Mannitol Salt Agar plates were used. Candida albicans and Aspergillus fumigatus spores were enumerated using Rose-Bengal Chloramphenicol Agar plates. Coliphages were cultivated using standard method described in ATCC manual. For all microbiological nutrient media were manufactured by HiMedia Laboratories, India unless mentioned otherwise.

\section{Aerosolization of test microbes A 6-jet Collison nebulizer (MESA} LABS, BGI) was used to aerosolize the test microbes into the test chamber. Dry air from a compressed air cylinder at a pressure of 10 psi was used to operate the nebulizer. The nebulizer produces bioaerosols of a $2-5 \mu \mathrm{m}$ diameter that allows them to float in the air present in the test chamber for a definite period. The length of the nebulization period varied depending on the type of experiment and microorganism, but typically ranged between 30-40 minutes.

D. Sampling of air for viable microbes The airborne survival of the test microbe and the activity of the air decontamination devices were determined by collecting the air from the chamber at the rate of 12.5 liter/min using SKC biosampler [54, filled with sterile buffer (1x Phosphate buffer saline, $\mathrm{pH}$ 7.2). Collected samples were analyzed to understand the quantity of viable microorganism present by diluting and plating them onto suitable growth media. The plated samples were incubated at $37 \pm 2{ }^{\circ} \mathrm{C}$ for bacteria and $25 \pm 2$ ${ }^{0} \mathrm{C}$ for fungal species and allowed to grow for 18-48 hours as mentioned in the ATCC/MTCC manual, individual colonies were enumerated, and the final concentration of the microbial load was calculated thereafter. For enumerating coliphages collected from the chamber, Double agar overlay method was used for subsequent plaque assay [55]. E. coli ATCC 15597 and E. coli ATCC 13706 were used as a host in plaque assays for MS2 and PhiX174, respectively. Plaques were counted after 24 hour incubation at $37 \pm 2{ }^{\circ} \mathrm{C}$. 


\section{${ }_{31}$ Acknowledgments}

332 333

\section{Field tests} of the entire process.

E. Spot experiments E. coli cells were grown in the standard medium. A known titre of cells were spotted onto a $25 \mathrm{~mm}^{2}$ surface and incubated for various time duration, both with and without electric field. Surfaces were resuspended in $500 \mu \mathrm{l}$ of sterile $1 \mathrm{X}$ PBS, which was then plated on standard agar plates to enumerate the microbes.

F. Limit of Detection Microbial enumeration is guided by two parameters, Limit of Detection (LOD) and Limit of Quantification (LOQ). For the present assays used to quantify the microbial load inside the test chamber, the LOD was $10 \mathrm{CFU}$ for bacterial and fungal load and $5 \mathrm{PFU}$ for viral load. However, LOD is always less than LOQ [56]. In many of our experimental analysis, post operating ZeBox device, the microbial detected numbers were in around LOD and hence, the exact LOQ was often indeterminant.

\section{G. SEM analysis of trapped microbes to decipher the mechanism of} kill 3D surfaces were stripped off from the electrode plates post operating the device against E.coli under challenge test under various time course, and treated with $2.5 \%$ glutaraldehyde in $0.1 \mathrm{M}$ phosphate buffer $(\mathrm{pH} 7.2$ ) for $24 \mathrm{hrs}$ at $4{ }^{\circ} \mathrm{C}$. The samples were dehydrated in series of graded ethanol solutions and subjected to critical point drying with CPD unit. The analyzed samples were mounted over the stud with double-sided carbon conductivity tape, and a thin layer of gold coat over the samples was done by using an automated sputter coater (EMITECK K550X Sputter Coater from EM Scientific Services) for 3 minutes and analyzed under Field Emission Scanning Electron Microscope (MERLIN Compact VP from M/s.Carl Ziess). The set parameters were: Working Distance $=5-6 \mathrm{~mm}$, EHT range $=2-4 \mathrm{kV}$, Range of Magnification $=70$ $\mathrm{KX}$, detectors $=\mathrm{SE} 2$ And InLens, machine under high vacuum.

H. Air sample collection A working tissue culture laboratory in a national stem cell research facility was chosen for study. This laboratory was situated in a building which had central airconditioning but the absence of a HEPA-enabled air handling unit resulted in frequent contamination of tissue culture samples. A handheld air sampler (SAS Super 100) was used, which could sample 100 liters of air per minute. Tryptic Soy Agar and Sabouraud dextrose agar plates were used to sample bacteria and fungi, respectively from the air. A fixed volume of air was sampled using the bio-sampler. Plates were placed in and removed from the bio-sampler in an aseptic manner. Plates were incubated at $25 \pm 2{ }^{\circ} \mathrm{C}$ (for fungal cultivation) and $37 \pm 2{ }^{\circ} \mathrm{C}$ (for bacterial cultivation) for 48 hours. Post-incubation, the number of colonies appeared were enumerated and converted to $\mathrm{CFU} / \mathrm{m}^{3}$ using statistical conversion provided by the manufacturer. Control plates were used to ensure the sterility

The authors acknowledge the Electron Microscopy Facility at Bangalore Life Science Cluster (C-CAMP, NCBS, inStem) for technical assistance in Scanning 
334 Electron microscopy imaging. Financial assistance was received from

Department of Biotechnology- Biotechnology Industry Research Assistance Council (DBT-BIRAC), Govt. of India under Small Business Innovation Research Initiative (SBIRI) (Grant no. BT/SBIRI1372/31/16), COVID-19 Consortium (Grant no. BT/COVID0025/01/20), and from Karnataka Innovation and Technology Society (KITS) Department of Electronics, IT, Bt and S\&T, Government of Karnataka, under Ideas2PoC Grant.

\section{Author contributions}

KSP, DK carried out experiments designed and supervised by SD, AG. DM, NH carried out theoretical and numerical work supervised by JA, GT. JV, KSR, SD, AG conceptualized and designed the technology. JV, AG secured and managed funding.

\section{Competing interests}

JA, GT declare no competing interests. SD is Director on Biomoneta board. The rest of the authors are, or were, employees of Biomoneta Research Private Limited, Bangalore, India 560065.

\section{References}

[1] Zhang Y, Mo J, Li Y, Sundell J, Wargocki P, Zhang J, Little JC, Corsi R, Deng Q, Leung MH, Fang L. Can commonly-used fan-driven air cleaning technologies improve indoor air quality? A literature review. Atmospheric Environment. 2011 Aug;45(26):4329-43.

[2] Liu G, Xiao M, Zhang X, Gal C, Chen X, Liu L, Pan S, Wu J, Tang L, Clements-Croome D. A review of air filtration technologies for sustainable and healthy building ventilation. Sustainable cities and society 2017 Jul:32:375-396.

[3] Afshari A, Ekberg L, Forejt L, Mo J, Rahimi S, Siegel J, Chen W, Wargocki P, Zurami S, Zhang J. Electrostatic Precipitators as an Indoor Air Cleaner-A Literature Review. Sustainability. 2020 Jan;12(21):8774.

[4] Simmons RB, Crow SA. Fungal colonization of air filters for use in heating, ventilating, and air conditioning (HVAC) systems. Journal of industrial microbiology. 1995 Jan;14(1):41-5.

[5] Kemp SJ, Kuehn TH, Pui DY, Vesley D, Streifel AJ. Filter collection efficiency and growth of microorganisms on filters loaded with outdoor air. ASHRAE Transactions. 1995 Jan:228-38.

[6] Maus R, Goppelsröder A, Umhauer H. Survival of bacterial and mold spores in air filter media. Atmospheric Environment. 2001 Jan;35(1):105-13. 
[7] Forthomme A, Joubert A, Andrès Y, Simon X, Duquenne P, Bemer D, Le Coq L. Microbial aerosol filtration: Growth and release of a bacteria-fungi consortium collected by fibrous filters in different operating conditions. Journal of aerosol science. 2014 Jun;72:32-46.

[8] Morisseau K, Joubert A, Le Coq L, Andres Y. Quantification of the fungal fraction released from various preloaded fibrous filters during a simulated ventilation restart. Indoor air. 2017 May;27(3):529-38.

[9] Stephens B, Novoselac A, Siegel JA. The effects of filtration on pressure drop and energy consumption in residential HVAC systems (RP-1299). HVAC\&R Research. 2010 May;16(3):273-94.

[10] Xu T, Lan CH, Jeng MS. Performance of large fan-filter units for cleanroom applications. Building and Environment. 2007 Jun;42(6):2299-304.

[11] Boelter KJ, Davidson JH. Ozone generation by indoor, electrostatic air cleaners. Aerosol science and technology. 1997 Jan 1;27(6):689-708.

[12] Gerone PJ, Couch RB, Keefer GV, Douglas RG, Derrenbacher EB, Knight V. Assessment of experimental and natural viral aerosols. Bacteriological reviews. 1966 Sep;30(3):576.

[13] Mainelis G. Collection of airborne microorganisms by electrostatic precipitation. Aerosol Science and Technology. 1999 Feb;30(2):127-44.

[14] Ghosh B, Lal H, Srivastava A. Review of bioaerosols in indoor environment with special reference to sampling, analysis and control mechanisms. Environment international. 2015 Dec;85:254-72.

[15] Zhang L, Luo J, Menkhaus TJ, Varadaraju H, Sun Y, Fong H. Antimicrobial nano-fibrous membranes developed from electrospun polyacrylonitrile nanofibers. Journal of membrane science. 2011 Mar;369(1-2):499-505.

[16] Mei Y, Yao C, Fan K, Li X. Surface modification of polyacrylonitrile nanofibrous membranes with superior antibacterial and easy-cleaning properties through hydrophilic flexible spacers. Journal of membrane science. 2012 Nov;417:20-7.

[17] Zhong Z, Xu Z, Sheng T, Yao J, Xing W, Wang Y. Unusual air filters with ultrahigh efficiency and antibacterial functionality enabled by $\mathrm{ZnO}$ nanorods. ACS applied materials \& interfaces. 2015 Sep;7(38):21538-44.

[18] Liu S, Zhao J, Ruan H, Wang W, Wu T, Cui W, Fan C. Antibacterial and anti-adhesion effects of the silver nanoparticles-loaded poly (L-lactide) fibrous membrane. Materials Science and Engineering: C. 2013 Apr;33(3):1176-82.

[19] Mei Y, Yao C, Li X. A simple approach to constructing antibacterial and anti-biofouling nanofibrous membranes. Biofouling. 2014 Mar;30(3):313-22. 
[20] Jeong SB, Heo KJ, Lee BU. Antimicrobial Air Filters Using Natural Sea Salt Particles for Deactivating Airborne Bacterial Particles. International journal of environmental research and public health. 2020 Jan;17(1):190.

[21] Xu P, Kujundzic E, Peccia J, Schafer MP, Moss G, Hernandez M, Miller SL. Impact of environmental factors on efficacy of upper-room air ultraviolet germicidal irradiation for inactivating airborne mycobacteria. Environmental science \& technology. 2005 Dec;39(24):9656-64.

[22] Ko G, First MW, Burge HA. Influence of relative humidity on particle size and UV sensitivity of Serratia marcescens and Mycobacterium bovis BCG aerosols. Tubercle and Lung Disease. 2000 Aug;80(4-5):217-28.

[23] Tang JW. The effect of environmental parameters on the survival of airborne infectious agents. Journal of the Royal Society Interface. 2009 Dec 6;6(suppl-6):S737-46.

[24] Memarzadeh F, Olmsted RN, Bartley JM. Applications of ultraviolet germicidal irradiation disinfection in health care facilities: effective adjunct, but not stand-alone technology. American journal of infection control. 2010 Jun;38(5):S13-24.

[25] Mamahlodi MT. Potential benefits and harms of the use of UV radiation in transmission of tuberculosis in South African health facilities. Journal of public health in Africa. 2019 May;10(1).

[26] Nardell EA, Bucher SJ, Brickner PW, Wang C, Vincent RL, Becan-McBride K, James MA, Michael M, Wright JD. Safety of upper-room ultraviolet germicidal air disinfection for room occupants: results from the Tuberculosis Ultraviolet Shelter Study. Public health reports. 2008 Jan;123(1):52-60.

[27] Zaffina S, Camisa V, Lembo M, Vinci MR, Tucci MG, Borra M, Napolitano A, Cannatà V. Accidental exposure to UV radiation produced by germicidal lamp: case report and risk assessment. Photochemistry and photobiology. $2012 \mathrm{Jul} ; 88(4): 1001-4$.

[28] Vaidya MU, Gangakhedkar GR, Shetty AN, Waghalkar PV. A rare occurrence of accidental exposure to UV radiation among operating theatre personnel. Indian journal of anaesthesia. 2020 Mar;64(3):230.

[29] Brickner PW, Vincent RL. Ultraviolet Germicidal Irradiation Safety Concerns: A Lesson from the Tuberculosis Ultraviolet Shelter Study Murphy's Law Affirmed. Photochemistry and photobiology. 2013 Jul;89(4):819-21.

[30] Kujundzic E, Matalkah F, Howard CJ, Hernandez M, Miller SL. UV air cleaners and upper-room air ultraviolet germicidal irradiation for controlling airborne bacteria and fungal spores. Journal of Occupational and Environmental Hygiene. 2006 Oct;3(10):536-46.

[31] Nakpan W, Yermakov M, Indugula R, Reponen T, Grinshpun SA. Inactivation of bacterial and fungal spores by UV irradiation and gaseous iodine treatment applied to air handling filters. Science of The Total Environment. 2019 Jun;671:59-65. 
[32] Bahri M, Haghighat F. Plasma-B ased Indoor Air Cleaning Technologies: The State of the Art Review. CLEAN-Soil, Air, Water. 2014 Dec;42(12):1667-80.

[33] Tang X, Feng F, Ye L, Zhang X, Huang Y, Liu Z, Yan K. Removal of dilute VOCs in air by post-plasma catalysis over Ag-based composite oxide catalysts. Catalysis today. 2013 Aug;211:39-43.

[34] Mamaghani AH, Haghighat F, Lee CS. Photocatalytic oxidation technology for indoor environment air purification: the state-of-the-art. Applied Catalysis B: Environmental. 2017 Apr;203:247-69.

[35] Chen F, Yang X, Mak HK, Chan DW. Photocatalytic oxidation for antimicrobial control in built environment: a brief literature overview. Building and environment. 2010 Aug;45(8):1747-54.

[36] Zhong L, Haghighat F. Photocatalytic air cleaners and materials technologies-abilities and limitations. Building and Environment. 2015 Sep;91:191-203.

[37] Mo J, Zhang Y, Xu Q, Zhu Y, Lamson JJ, Zhao R. Determination and risk assessment of by-products resulting from photocatalytic oxidation of toluene. Applied Catalysis B: Environmental. 2009 Jul;89(3-4):570-6.

[38] Wei K, Zou Z, Yao M. Charge levels and Gram ( \pm ) fractions of environmental bacterial aerosols. Journal of aerosol science. 2014 Aug; $74: 52-62$.

[39] Shen F, Kai W, Yao M. Negatively and positively charged bacterial aerosol concentration and diversity in natural environments. Chinese Science Bulletin. 2013 Sep;58(26):3169-76.

[40] Tosun I, Uner D, Ozgen C. Critical Reynolds number for Newtonian flow in rectangular ducts. Industrial \& engineering chemistry research. 1988 Oct;27(10):1955-7.

[41] Kundu P.K., Cohen I.M., Hu H.H. Fluid Mechanics. Academic press, London (pp. 516); 2008.

[42] Kassab AS, Ugaz VM, King MD, Hassan YA. High resolution study of micrometer particle detachment on different surfaces. Aerosol Science and Technology. 2013 Apr;47(4):351-60.

[43] Soltani M, Ahmadi G. On particle adhesion and removal mechanisms in turbulent flows. Journal of Adhesion Science and Technology. 1994 Jan;8(7):763-85.

[44] Mainelis G, Willeke K, Baron P, Reponen T, Grinshpun SA, Górny RL, Trakumas S. Electrical charges on airborne microorganisms. Journal of Aerosol Science. 2001 Sep;32(9):1087-110.

[45] Lee SA, Willeke K, Mainelis G, Adhikari A, Wang H, Reponen T, Grinshpun SA. Assessment of electrical charge on airborne microorganisms by a new bioaerosol sampling method. Journal of occupational and environmental hygiene. 2004 Mar;1(3):127-38. 
[46] Hugo WB. The mode of action of antibacterial agents. Journal of Applied Bacteriology. 1967 Apr;30(1):17-50.

[47] Denyer SP. Mechanisms of action of antibacterial biocides. International biodeterioration \& biodegradation. 1995 Oct;36(3-4):227-45.

[48] McDonnell G, Russell AD. Antiseptics and disinfectants: activity, action, and resistance. Clinical microbiology reviews. 1999 Jan;12(1):147-79.

[49] Tischer M, Pradel G, Ohlsen K, Holzgrabe U. Quaternary ammonium salts and their antimicrobial potential: targets or nonspecific interactions?. ChemMedChem. 2012 Jan;7(1):22-31.

[50] Kotnik T, Rems L, Tarek M, Miklavčič D. Membrane electroporation and electropermeabilization: mechanisms and models. Annual review of biophysics. 2019 May;48:63-91.

[51] Jose S, Phadke KS, Venkatraman J, Krishna B, Sampath S, Datta S, Nagaraj S, Ghatak A. Trap and kill of environmental microbes: Validation of a novel decontamination technology in Hospital ICU setting. medRxiv. 2020 Jan.

[52] Li DT, Samaranayake LP, Leung YY, Neelakantan P. Facial protection in the era of COVID-19: A narrative review. Oral diseases. 2020 Jun 7.

[53] Coulliette AD, Perry KA, Fisher EM, Edwards JR, Shaffer RE, Noble-Wang J. MS2 coliphage as a surrogate for 2009 pandemic influenza A (H1N1) virus (pH1N1) in surface survival studies on N95 filtering facepiece respirators. Journal of the International Society for Respiratory Protection. 2014 Jan;21(1):14.

[54] Li J, Leavey A, Wang Y, O'Neil C, Wallace MA, Burnham CA, Boon AC, Babcock H, Biswas P. Comparing the performance of 3 bioaerosol samplers for influenza virus. Journal of aerosol science. 2018 Jan;115:133-45.

[55] Kropinski AM, Mazzocco A, Waddell TE, Lingohr E, Johnson RP. Enumeration of bacteriophages by double agar overlay plaque assay. InBacteriophages 2009 (pp. 69-76). Humana Press.

[56] Armbruster DA, Pry T. Limit of blank, limit of detection and limit of quantitation. The clinical biochemist reviews. 2008 Aug;29(Suppl 1):S49. 


\section{${ }_{530}$ Supplementary information}

S1. Range of microbes. To analyze microbe's motion in laminar flow between electrode-plates we adopt the following approximations: (1) The flow is identical to that between infinitely wide plates, which is justified because $W / H \gg 1$, where $H$ is the gap between electrode-plates and $W$ is their width (perpendicular to the flow direction); (2) The flow is fully developed, which is justified because $L / H \gg 1$, where $L$ is the length of electrode-plates along the flow direction; (3) The microbes move with the flow except when electric force acts on them, which is justified because the Stokes number of microbes (a measure of its inertial response to changes in the flow) is $\ll 1$; (4) Self weight of microbes is negligible compared to the electric forces acting on them, because of their extremely small size $(<5 \mu \mathrm{m})$.

The orientation of our coordinate system is shown in figure 1. A steady, unidirectional, incompressible, fully-developed flow is governed by [1]:

$$
\begin{array}{cl}
\frac{d u}{d x}=0 & \text { (Mass conservation) } \\
\frac{d p}{d x}=\mu \frac{d^{2} u}{d z^{2}} & \text { (Momentum conservation) }
\end{array}
$$

where $u$ is the flow velocity along $X$ direction, $p$ is pressure and $\mu$ is dynamic viscosity of the fluid. Since, subject to our assumptions, $u$ depends only on $z$, mass conservation in Eqn. 1 is automatically satisfied. Since the flow is induced by imposing a pressure difference between the ends of the electrode-plates, the pressure gradient $d p / d x$ is a constant. Therefore, momentum conservation in Eqn. 2 is satisfied if $u$ is a quadratic function of $z$. We assume it to be of the form, $u=A z(H-z)$, because this automatically satisfies the no-slip boundary condition on the electrode-plates located at $z=0, H$. The constant $A$ is determined by computing the volumetric flow rate and equating it to the known value $Q, W \int_{0}^{H} d z u=Q$, which yields:

$$
u(z)=\frac{6 Q}{W H^{3}} z(H-z)
$$

where $Q$ is the volumetric flow rate of air between the electrode-plates. The flow velocity varies only along $Z$ direction, being maximum midway between the plates and zero at the plates themselves (no-slip condition). Because the Reynolds number of microbe's motion is $\ll 1$, due to its small size and small speeds, only Stokes drag force is exerted by the ambient fluid, $F_{\text {drag }}=6 \pi \mu w a$, where $\mu$ is the dynamic viscosity of air. We have assumed that the microbe can be approximated by an equivalent sphere of radius $a$. The drag counterbalances the electric force on the microbe, $F_{\text {electric }}=q E$, where $q$ is the surface charge on the microbe and $E$ is the strength of the applied electric field. Equating the two forces yields for the settling velocity:

$$
w=\frac{q E}{6 \pi \mu a}
$$

While drifting towards the electrode-plate the microbe also travels a distance $R$ in the flow direction, which we call its "range", refer figure 1] If $z_{0}$ 
is the initial distance of the microbe from the attracting electrode-plate at its entrance $x=0$, then the time $T$ needed for the microbe to hit the electrode-plate is, $T=z_{0} / w$. After time $t$, the vertical location of the microbe initially located at $z_{0}$ will be $z=z_{0}-w t$. Then, from eqn. 3 the microbe's streamwise speed at that time will be $u\left(z_{0}-w t\right)$. The microbe will hit the electrode-plate in time $T=z_{0} / w$ (assuming sufficient plate length). Therefore the range of the microbe beginning at location $z_{0}$ is given by $R\left(z_{0}\right)=\int_{0}^{T} d t u\left(z_{0}-w t\right)$. Changing the integration variable to $z=z_{0}-w t$ transforms the integral to: $R\left(z_{0}\right)=w^{-1} \int_{0}^{z_{0}} d z u(z)$. Substituting from eqn. 3 and integrating yields:

$$
R\left(z_{0}\right)=\frac{6 Q z_{0}^{2}}{W H^{3} w}\left(\frac{H}{2}-\frac{z_{0}}{3}\right), \quad 0 \leq z_{0} \leq H
$$

The microbe that is farthest from the attracting electrode-plate, i.e. at $z_{0}=H$, has the maximum range:

$$
R_{\max }=\frac{Q}{W w}
$$

All the microbes entering ZeBox will hit the electrode-plate if its length is not less than the maximum range of the microbes, i.e. if $L \geq R_{\max }$. Eq 5 for the range is visualized more easily if we divide it through by $R_{\max }, \mathrm{Eq} 6$, and rewrite it in the following dimensionless form:

$$
\frac{R}{R_{\max }}=6\left(\frac{z_{0}}{H}\right)^{2}\left(\frac{1}{2}-\frac{\left(z_{0} / H\right)}{3}\right), \quad 0 \leq \frac{z_{0}}{H} \leq 1
$$

Assuming that all the trapped microbes are killed, Eq 7 plotted in supplementary figure 7 completely determines the microbicidal efficiency of ZeBox, as per the present model. Here, "microbicidal efficiency" is defined as the fraction of microbes entering electrode-plates that hit it and are inactivated, assuming a uniform distribution at the entrance. Using supplementary figure 7, the microbicidal efficiency is found as follows. We first compute $L / R_{\max }$ given the operating parameters. If $L / R_{\max } \geq 1$, then the microbicidal efficiency is of course $100 \%$. Otherwise, we locate its value on the vertical axis of supplementary figure 7 and using the curve find the corresponding value on the horizontal axis, which gives the microbicidal efficiency. Therefore, $L / R_{\max }$ alone determines the microbicidal efficiency of ZeBox according to the present model.

S2. Microbicidal efficiency of ZeBox. A microbe in an ionic solution is surrounded by a diffuse double layer of ions of molecular dimensions. The Debye length $\left(\kappa^{-1}\right)$, which is a measure of the thickness of the double layer, lies in the range: $1<\kappa^{-1}<10 \mathrm{~nm}[2$. Since the microbe's size $a \sim 1 \mu \mathrm{m}$, $\kappa a \gg 1$ for microbes. The magnitude of the measured zeta potential $(\zeta)$ of microbes in phospate-buffer solution lies in the range 1 to $30 \mathrm{mV}$. Considering the worst-case-scenario, we may take $\zeta=1 \mathrm{mV}$ and $\kappa a=100$. The number of elementary charges $n$ on the microbe may then be estimated as [2]: 


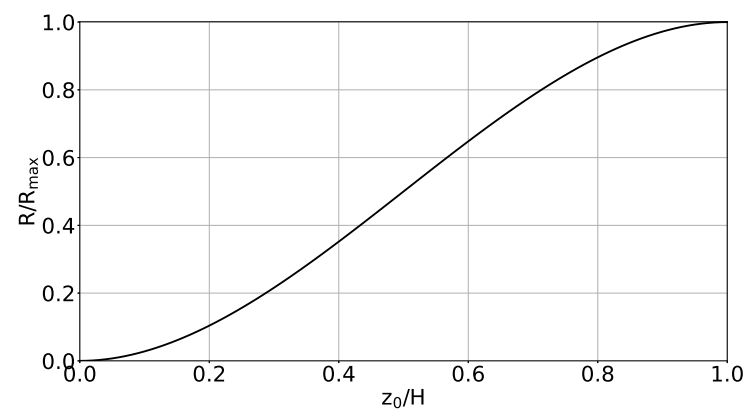

Supplementary figure 7: Range of a microbe. A microbe initially at distance $z_{0} / H$ from the attracting electrode-plate hits it at a distance $R / R_{\max }$.

$$
n \approx \frac{4 \pi \epsilon_{r} \epsilon_{0} \zeta a(1+\kappa a)}{e}
$$

where $\epsilon_{r}$ is the dielectric constant of the solution, $\epsilon_{0}=8.9 \times 10^{-12} \mathrm{~F} / \mathrm{m}$ is the permittivity of vaccuum and $e=1.6 \times 10^{-19} \mathrm{C}$ is the magnitude of the elementary charge. Eqn. 8 is derived from the linearized Poisson-Boltzmann equation governing the variation of electric potential due to distribution of ions in the diffuse layer surrounding a charged sphere; the linearization is a consequence of the Debye-Hückel approximation which is applicable when zeta potential is small (compared to $\sim 25 \mathrm{mV}$ at $25{ }^{\circ} \mathrm{C}$ ) [2]. For a measured dielectric constant of 78.5 for the buffer solution, Eqn. 8 reveals $n>5000$ elementary charges. Even allowing for an order-of-magnitude error and assuming $n>500$ instead, Eqn. 4 yields a settling velocity of $w>7 \mathrm{~cm} / \mathrm{s}$, for $E=3 \mathrm{kV} / \mathrm{cm}$ in air.

Since the flow rate between a pair of electrode-plates is $Q<3 \mathrm{cfm}$ and the electrode-plate width $W=10.9 \mathrm{~cm}$, Eqn. 6 shows that $R_{\max }<19 \mathrm{~cm}$. In comparison, ZeBox employs $30 \mathrm{~cm}$ long electrode-plates. Although in theory it implies $100 \%$ microbicidal efficiency for ZeBox, the present model is only approximate because it does not account for the effects of possible turbulence in the flow and slippage of microbes on the surface. In reality, as mentioned in the Results section, we obtain 83-99 \% microbicidal efficiency for ZeBox as deduced from the measured microbial load reduction.

\section{References}

[1] Kundu P.K., Cohen I.M., Hu H.H. Fluid Mechanics. Academic press, London (pp. 516); 2008.

[2] Hiemenz P. C., Rajagopalan R. Principles of Colloid and Surface Chemistry. 